\title{
Application of Angio Optical Coherence Tomography and Flash Electroretinogram in the Study of Retinal Morphology and Function in High Myopia
}

\author{
Mengdan Cao \\ Central South University \\ Jinxiang Luo \\ Aier Glaucoma Research Institute, Changsha Aier Eye Hospital \\ Junyi Ouyang \\ Aier Glaucoma Research Institute, Changsha Aier Eye Hospital \\ Fen Nie \\ Central South University \\ Xuanchu Duan ( $\nabla$ duanxchu@126.com ) \\ Aier Glaucoma Research Institute, Changsha Aier Eye Hospital
}

\section{Research Article}

Keywords: High myopia, OCTA, FERG, Macula

Posted Date: December 1st, 2021

DOl: https://doi.org/10.21203/rs.3.rs-1057602/v1

License: (c) (i) This work is licensed under a Creative Commons Attribution 4.0 International License. Read Full License 


\section{Abstract}

Purpose: Using optical coherence tomography (OCTA) and flash electroretinography (F-ERG), we investigated changes to the retinal micromorphology and the overall function of the retina in eyes with different degrees of high myopia.

Design: Case-control study.

Participants: A total of 64 cases of 118 eyes with axial high myopia with diopters ranging from --6.0 D to $-15.0 D$ were included in the study. The individuals were 18-40 years old.

Methods: Subjects were divided into three groups according to the axial length ( $A L)$ of the eye and the spherical equivalent (SE)of the lens: the high myopia group (AL: $\leq 26 \mathrm{~mm}$ and SE: $-6.0 \mathrm{D}$ to $-9.0 \mathrm{D}$ ) with 32 eyes, the ultra-high myopia group (AL: 26-28 mm and SE: -9.25D to -12.0D) with 43 eyes, and the extremely high myopia group (AL: $\geq 28 \mathrm{~mm}$ and SE: $-12.25 \mathrm{D}$ to $-15.0 \mathrm{D}$ ) with 43 eyes. We used the OCTA measurements macular foveal retinal thickness (MRT), foveal avascular zone (FAZ), and superficial macular and deep retinal microvascular density, and the F-ERG measurements retinal dark-adaptation a-wave and b-wave, oscillation potential (OP) wave, light adaptation a-wave and b-wave, and 30- $\mathrm{Hz}$ flicker light amplitudes, which represent the functional state of the retina.

Results: The differences in retinal thickness in the macular area between the three groups were only statistically significant within $1 \mathrm{~mm}$ of the fovea $(P=0.006)$. MRT was positively correlated with $A L(r=0.278, P=0.002)$ and negatively correlated with SE $(R=-0.200, P=0.031)$. The difference in the FAZ area between the three groups was also statistically significant $(P=0.036)$, and FAZ was negatively correlated with $A L(r=-0.377, p<0.001)$ and positively correlated with SE $(r=0.192, P<0.5)$. Both the superficial and deep blood flow density of the macular fovea were positively correlated with $A L(p<0.001)$. The superficial parafoveal blood flow density was negatively correlated with $A L(r=-0.280, P=0.002)$ but positively correlated with $S E(R=0.254, P=0.006)$. The overall blood flow density of the deep retina, the parafoveal blood flow density, and the blood flow density around the fovea were negatively correlated with $A L(p<0.001)$ and positively correlated with myopic SE $(\mathrm{p}<0.001)$. The dark-adaptation b-wave, maximum comprehensive response a-wave and bwave, OP wave, bright-adaptation a-wave and b-wave, and $30-\mathrm{Hz}$ flicker amplitudes of the retina were negatively correlated with $\mathrm{AL}$ and positively correlated with SE. The amplitudes of light adaptation a-wave and b-wave were[1] negatively correlated with the foveal avascular density $(p<0.001)$, and the average amplitude of the OP wave was positively correlated with the superficial retinal avascular density $(p<0.001)$.

Conclusions: Before obvious pathological changes or central vision damage, the morphology and function of the macular area show subtle changes in patients with high myopia. As the degree of myopia increases and the axis of the eye increases, the activity of the cone cells in high myopia eyes decreases and the microvascular circulation in the inner retina and function of inner layer cells, such as non-proa cells, are affected. The changes of retinal dark adaptation are more sensitive than those of bright adaptation, which indicates that retinal rod cell damage may be earlier than cone cell damage in high myopia. Therefore, we believe that the combined use of OCTA and F-ERG can help in the early diagnosis and monitoring of patients at a high risk of myopia and to guide their clinical treatment.

Because the p-value is clearly provided, the term "significantly" is not necessary (removed to reduce wordiness).

\section{Background}

Myopia is a common ocular disease in Asian populations[1], and it is [1] also the most prevalent ocular disease among adolescents. The prevalence of myopia is as high as $80 \%$ in Asia, compared with $25 \%$ in other regions[2-4]. High myopia refers to eyes with a spherical equivalent (SE) of $\leq-6.0 \mathrm{D}$ or/and an axial length (AL) [2] of $\geq 26 \mathrm{~mm}$ and is characterized by a gradual extension of the eye axis and gradual degeneration of the posterior pole. With the continuous progression of high myopia, further stretching of the eye axis causes a series of pathological changes to the fundus, such as optic disc

Page 2/18 
myopic arc, posterior scleral staphyloma, peripheral retinal degeneration, Fuchs spot, and macular membrane [5-6], and it may also be complicated by cataracts, glaucoma, and retinal detachment, etc. [7-9]. As a result of these complications, one-third of people with high myopia will develop severe visual impairment during their lifetime. The risk of severe visual impairment increases significantly with the extension of the refractive power or AL [3] [10]. Therefore, research into high myopia is particularly important.

To date, research on the morphology of the retina in people with high myopia has mainly focused on analyses of the retinal thickness, retinal nerve fiber layer thickness, choroidal thickness, and changes to the retinal blood flow and choroidal blood flow[4] using optical coherence tomography (OCT/OCTA). Research has shown that both the decreased thickness of the retina, retinal nerve fiber layer, and choroid, and the avascular density of the retina and choroid [5] are closely related to changes in AL and myopic diopter[10]. Current research into retinal function involves examinations of retinal light sensitivity and retinal electrophysiology. According to previous findings, compared with emmetropic patients, both the retinal photosensitivity[11-12] and electroretinogram (ERG) amplitude [13-14] of highly myopic patients are decreased, and these changes are also related to changes in the AL and myopic diopter.

However, there have been few studies on the relationship between the structure and function of the retina in high myopia eyes. In our research, we measured the retinal thickness at the center of the macula, the macular foveal avascular area, and the density of microvessels in the macular area using OCTA, and the retinal function was measured by flash ERG (FERG). We aimed to provide guidance for the exploration and treatment of high myopia pathogenesis by analyzing the connection between the structure and function of the retina.

\section{Methods}

Subjects: The study protocol adhered to the tenets of the Declaration of Helsinki and was approved by the institutional review board of the Changsha Aier Ophthalmology Refractive Clinic. The participants were informed of the study objectives, and signed informed consent was obtained from all participants. All 64 patients (118 eyes) with different degrees of high myopia who attended the Changsha Aier Ophthalmology Refractive Clinic were prospectively and consecutively recruited from February to August 2019 and ranged in age from 18 to 40 years old. All subjects underwent a complete ophthalmic examination to include naked vision (standard logarithmic eye chart), best-corrected visual acuity (comprehensive refractometer, NIDER, Japan), slit-lamp examination, intraocular pressure (IOP, non-contact tonometer, Canon TX-20, Japan), AL (IOL Master intraocular lens biometer, Zeiss 500, Germany), OCTA, and F-ERG examinations. Additionally, a detailed medical history was also recorded for each subject.

The selected cases were divided into three groups according to the refractive power and length of the eye axis: (1) High myopia group ( $\mathrm{AL} \leq 26 \mathrm{~mm}$ and $-6.0 \mathrm{D} \leq \mathrm{SE} \leq-9.0 \mathrm{D}$ ) with 32 eyes; (2) Ultra-high myopia group (AL 26-28 mm and -9.25D $\leq \mathrm{SE} \leq-12.0 \mathrm{D}$ ) with 43 eyes; (3) Extremely high-myopia group ( $\mathrm{AL} \geq 28 \mathrm{~mm}$ and $-12.25 \mathrm{D} \leq \mathrm{SE} \leq-15.0 \mathrm{D}$ ) with 43 eyes.

The following inclusion criteria were used: age in the range of 18-40 years, normal eye position, refractive range of $-6.0 \mathrm{D}$ to $-15.0 \mathrm{D}$, best corrected vision $\geq 0.8$, intraocular pressure $\leq 21 \mathrm{mmHg}$, refractive interstitial transparency, and OCTA image signal $\geq 7$. While the exclusion criteria included cylinder power greater than 2.0D; previous history of eye trauma or eye surgery; history of other eye disease or family history of glaucoma; history of systemic diseases, such as hypertension, diabetes, rheumatism, etc.; history of taking drugs that may affect blood circulation within a week. Evaluators were blind to all other patient and ocular data, and eyes were excluded from the study analyses if a consensus could not be reached. The right eyes were selected for the analyses.

Angio Optical Coherence Tomography (OCTA): [1] [2] The OCTA (OptovueRTVue-XR Avanti, Optovue Inc., Fremont, California, USA) "retina-scan" mode was used to obtain retinal vascular density information (Figure 1), and the same 
operator used an AngioVue instrument to obtain clear fundus images. The system automatically scanned areas of $3 \times 3$ $\mathrm{mm}$ and $6 \times 6 \mathrm{~mm}$ centered on the macular fovea. After scanning, the system automatically divided the 3D images into the superficial retinal capillary network and the En-Face layered image of the deep retinal capillary network according to structure.

Flash electroretinogram: F-ERG (ESPION, USA) is an objective examination that reflects the overall function of the retina. The subject was positioned in a dark room for 30 minutes, their skin was cleaned under dark red light, and electrodes were placed on the skin. The ground electrode was placed in the middle of the forehead, the reference electrode was placed on the lateral canthus of both eyes, and surface anesthetic was applied. The corneal contact electrode was applied, and the subject was instructed to keep looking at the red fixation point in the center of the Ganzfeld stimulator and to try not to blink during the stimulation. We then initiated $0.01 \mathrm{~cd} \cdot \mathrm{s} / \mathrm{m}^{2}, 3.0 \mathrm{~cd} \cdot \mathrm{s} / \mathrm{m}^{2}$, and $10.0 \mathrm{~cd} \cdot \mathrm{s} / \mathrm{m}^{2}$ brightness flash inspections in sequence, after which the weak red light was turned off for 5 minutes to allow the eyes to adapt to the dark. In dark conditions, the rod cell response, maximum comprehensive response, and oscillation potential (OP) were recorded. After completion, the light was turned on, and the light-adaptation step was started. The subject was emersed in light under a background of $3.0 \mathrm{~cd} \cdot \mathrm{s} / \mathrm{m}^{2}$ and allowed to adapt for 10 minutes. The cone cell response and the $30-\mathrm{Hz}$ scintillation light response were recorded in sequence. All responses were stimulated twice, and the computer automatically superimposed the average value to eliminate the abnormal artifacts of the original waveform caused by eye movement, contact lens bubbles, blinking, or eye muscle twitching. [4] For the standard measurement of the peak times and amplitude parameters of each response waveform, we adopted ISCEV (International Association for Visual Electrophysiology) standard measurements.

Statistical analysis of data: SPSS25.0 software was used for data analysis. The results are expressed as the mean \pm standard deviation (SD), and five reaction indicators of gender, age, AL, SE, macular retinal thickness, foveal avascular zone (FAZ), blood vessel density in each macular region, and F-ERG of the three groups of subjects were used in the analyses. Data analysis was performed on the parameters and the relationship between them. Differences between groups were analyzed by variance analysis (one-way ANOVA method), and pairwise comparisons between groups were performed by Tukey's analysis. Pearson's correlation analysis was used to determine the correlations between two datasets. We set the $P$ value for significance at $<0.05$.

\section{Results}

We inclu[1] ded 118 eyes with high myopia in the study, and the cases were grouped, according to the lens SE, into the high myopia, ultra-high myopia, and extremely high myopia group[2] s. There were no significant differences in gender or age among the three groups $(P>0.05)$, and the differences between $S E$ and $A L[3]$ were statistically significant $(P<0.001)$ (Table 1). Pearson's correlation analysis showed that SE was closely related to AL $(R=-0.706, P<0.001)$. As the degree of myopia increased, the $A L$ increased, and SE and AL were negatively correlated.

Table 1 Basic information on the three groups 
High myopia group Ultra-high myopia group Extremely high myopia group $\mathrm{P}$

\begin{tabular}{lllll}
\hline Number of eyes & 32 & 43 & 43 & - \\
\hline Gender (M:F) & $9: 23$ & $11: 32$ & $19: 24$ & 0.149 \\
\hline Age (years) & $26 \pm 5.00$ & $24.05 \pm 4.1$ & $27.07 \pm 7.49$ & 0.053 \\
\hline Axial length [4] (mm) & $25.49 \pm 0.62$ & $27.06 \pm 0.57$ & $28.63 \pm 0.70$ & 0.000 \\
\hline Spherical equivalent (SE) & $-7.93 \pm 1.22$ & $-10.00 \pm 1.59$ & $-12.39 \pm 2.55$ & 0.000
\end{tabular}

OCTA assessment of retinal microvascular morphologic changes in people with high myopia

\section{Comparison of retinal thickness in macular area}

The average retinal thickness of the macular fovea $(1 \mathrm{~mm})$, parafovea (1-3 mm), and surrounding areas (3-6 mm) of the three groups are shown in Table 2. Only the retinal thickness of the macular fovea (macular foveal retinal thickness, MRT) was significantly different among groups $(P=0.006)$, and it positively correlated with $A L(r=0.278, P=0.002)$ and negatively correlated with myopic SE $(R=-0.200[5], P=0.031)$.

\section{Comparison of fovea avascular area}

The average FAZ values of the three groups are shown in Table 2. The differences in FAZ between the three groups were statistically significant $(P<0.05)$. FAZ was negatively correlated with $A L(R=-0.377, p<0.001)$ and positively correlated with $S E(R=0.192[6], P<0.05)$.

Table 2 Correlation between retinal thickness/FAZ and AL/SE in macular area

\begin{tabular}{|c|c|c|c|c|c|c|c|c|}
\hline \multirow[t]{2}{*}{ Location/Group } & \multirow{2}{*}{$\begin{array}{l}\text { High } \\
\text { myopia } \\
\text { group }\end{array}$} & \multirow[t]{2}{*}{$\begin{array}{l}\text { Ultra-high } \\
\text { myopia group }\end{array}$} & \multirow[t]{2}{*}{$\begin{array}{l}\text { Extremely high } \\
\text { myopia group[7] }\end{array}$} & \multirow[t]{2}{*}{$\mathrm{P}$} & \multicolumn{2}{|c|}{$\begin{array}{l}\text { Relationship } \\
\text { with AL }\end{array}$} & \multicolumn{2}{|c|}{$\begin{array}{l}\text { Relationship } \\
\text { with SE }\end{array}$} \\
\hline & & & & & $\mathrm{R}$ & $P$ & $\mathrm{R}$ & $P$ \\
\hline $1 \mathrm{~mm}$ & $243.6 \pm 22.3$ & $246.9 \pm 12.9$ & $259.0 \pm 27.6$ & 0.006 & $.278^{\star *}$ & 0.002 & $-.200^{*}$ & 0.031 \\
\hline $1-3 \mathrm{~mm}$ & $306.4 \pm 48.6$ & $313.2 \pm 10.5$ & $306.3 \pm 21.4$ & 0.473 & -.075 & 0.425 & 0.059 & 0.532 \\
\hline $3-6 \mathrm{~mm}$ & $255.9 \pm 68.6$ & $272.3 \pm 12.6$ & $267.7 \pm 11.8$ & 0.168 & -.005 & 0.958 & -.089 & 0.343 \\
\hline FAZ & $0.29 \pm 0.14$ & $0.27 \pm 0.10$ & $0.22 \pm 0.11$ & 0.036 & $-.377^{* *}$ & 0.000 & $.192^{*}$ & 0.037 \\
\hline
\end{tabular}

** At 0.01 level (two-tailed), the correlation was significant. * At 0.05 level (two-tailed), the correlation was significant.

FAZ area: hollow non-irrigation area

\section{Comparison of blood flow density in macular area}

The blood flow densities in the macular areas of the three groups are shown in Table 3. The superficial foveal blood flow density was positively correlated with $\mathrm{AL}(\mathrm{R}=0.392, \mathrm{p}<0.001)$ and negatively correlated with myopic $\mathrm{SE}(\mathrm{R}=-0.227[8][9]$, $P=0.014$ ). The parafoveal blood flow density and the TSNI[10] zone blood flow density were significantly different (both $P<0.05)$ among the groups, and they negatively correlated with $A L(R=-0.280, P=0.002)$ and positively correlated with myopic SE $(R=0.254,[11] P=0.006[12])$.

The blood flow density in the deep macular fovea showed significant differences among groups $(P<0.05)$, and it positively correlated with $A L(R=-0.366, P<0.001)$ but did not correlate with myopic $S E(P>0.05)$. 
Table 3 Blood flow density in the macular area and its correlation with AL and SE

\begin{tabular}{|c|c|c|c|c|c|c|}
\hline \multirow[t]{2}{*}{ Location/Group } & \multirow{2}{*}{$\begin{array}{l}\text { High } \\
\text { myopia } \\
\text { group }\end{array}$} & \multirow[t]{2}{*}{$\begin{array}{l}\text { Ultra-high } \\
\text { myopia group }\end{array}$} & \multirow[t]{2}{*}{$\begin{array}{l}\text { Extremely high } \\
\text { myopia group }\end{array}$} & \multirow[t]{2}{*}{$P$} & $\begin{array}{l}\text { Relationship } \\
\text { with } A L\end{array}$ & $\begin{array}{l}\text { Relationship } \\
\text { with SE[13] }\end{array}$ \\
\hline & & & & & $P$ & $\mathrm{R}$ \\
\hline
\end{tabular}

Superficial retina (\%)

\begin{tabular}{|c|c|c|c|c|c|c|c|c|}
\hline Fovea & $19.90 \pm 8.93$ & $21.45 \pm 5.39$ & $24.85 \pm 8.30$ & 0.027 & $.392^{\star *}$ & 0.000 & $-.227^{*}$ & 0.014 \\
\hline Para-fovea & $52.85 \pm 4.20$ & $51.95 \pm 3.57$ & $50.20 \pm 3.57$ & 0.001 & $-.280^{\star \star}$ & 0.002 & $.254^{* *}$ & 0.006 \\
\hline $\mathrm{T}$ & $52.80 \pm 4.72$ & $52.25 \pm 3.49$ & $49.60 \pm 4.01$ & 0.000 & $-.336^{* *}$ & 0.000 & $.306^{* *}$ & 0.001 \\
\hline S & $53.92 \pm 4.58$ & $53.40 \pm 3.94$ & $51.57 \pm 3.67$ & 0.002 & $-.249^{\star *}$ & 0.007 & $.237^{\star \star}$ & 0.010 \\
\hline $\mathrm{N}$ & $51.91 \pm 4.10$ & $50.60 \pm 4.59$ & $49.19 \pm 4.73$ & 0.034 & $-.234^{*}$ & 0.011 & $.223^{*}$ & 0.015 \\
\hline I & $52.75 \pm 5.09$ & $51.53 \pm 4.18$ & $50.45 \pm 4.41$ & 0.028 & -.176 & 0.056 & 0.139 & 0.133 \\
\hline \multicolumn{9}{|c|}{ Deep retina (\%) } \\
\hline Fovea & $37.44 \pm 9.50$ & $38.44 \pm 6.56$ & $42.05 \pm 9.16$ & 0.036 & $.366^{\star *}$ & 0.000 & -.169 & 0.068 \\
\hline Para-fovea & $56.12 \pm 4.57$ & $55.72 \pm 4.09$ & $52.86 \pm 4.43$ & 0.002 & $-.321^{\star *}$ & 0.000 & $.347^{* \star}$ & 0.000 \\
\hline $\mathrm{T}$ & $57.70 \pm 4.45$ & $56.75 \pm 3.81$ & $54.08 \pm 4.17$ & 0.001 & $-.377^{* *}$ & 0.000 & $.397^{* \star}$ & 0.000 \\
\hline S & $55.33 \pm 5.44$ & $55.15 \pm 4.64$ & $51.73 \pm 5.72$ & 0.008 & $-.314^{\star *}$ & 0.001 & $.379^{\star \star}$ & 0.000 \\
\hline $\mathrm{N}$ & $57.20 \pm 4.57$ & $56.76 \pm 4.48$ & $54.58 \pm 4.15$ & 0.004 & $-.271^{\star *}$ & 0.003 & $.276^{\star \star}$ & 0.002 \\
\hline I & $53.82 \pm 5.70$ & $54.22 \pm 4.93$ & $51.05 \pm 4.91$ & 0.012 & $-.215^{*}$ & 0.020 & $.227^{*}$ & 0.014 \\
\hline
\end{tabular}

** At 0.01 level (double tail), the correlation was significant. * At 0.05 level (double tail), the correlation was significant.

Whole: total blood flow density; Fovea: foveal blood flow density; Parafovea: parafoveal blood flow density; T: temporal blood flow density; S: upper blood flow density; N: nasal blood flow density; I: lower blood flow density

\section{F-ERG reaction}

The amplitude and peak response time waveform differences and correlation with AL and SE are provided in Tables 4 and 5. The amplitude of dark adaptation 3.0 a-wave, dark adaptation 3.0 b-wave, bright adaptation 3.0 a-wave, and bright adaptation 3.0 b-wave were significantly different between the groups $(P<0.05)$ and were negatively correlated with $A L(P$ $<0.05)$ and positively correlated with myopic SE $(P<0.05)$. There was a significant difference between the group's peak times of dark adaptation 3.0 a-wave, which was negatively correlated with myopic SE.

Table 4 Differences in the amplitude of response waveforms and correlation with AL and SE in the F-ERG 


\begin{tabular}{|c|c|c|c|c|c|c|c|c|}
\hline \multirow[t]{2}{*}{$\begin{array}{l}\text { Amplitude } \\
(\mu \mathrm{V}[14])\end{array}$} & \multirow[t]{2}{*}{$\begin{array}{l}\text { High myopia } \\
\text { group }\end{array}$} & \multirow[t]{2}{*}{$\begin{array}{l}\text { Ultra-high } \\
\text { myopia group }\end{array}$} & \multirow{2}{*}{$\begin{array}{l}\text { Extremely } \\
\text { high myopia } \\
\text { group }\end{array}$} & \multirow[t]{2}{*}{$P$} & \multicolumn{2}{|c|}{$\begin{array}{l}\text { Relationship } \\
\text { with AL }\end{array}$} & \multicolumn{2}{|c|}{$\begin{array}{l}\text { Relationship } \\
\text { with SE }\end{array}$} \\
\hline & & & & & $\mathrm{R}$ & $P$ & $\mathrm{R}$ & $P$ \\
\hline $\begin{array}{l}\text { Dark } \\
\text { adaptation } 3.0 \\
\text { a-wave }\end{array}$ & $267.54 \pm 88.61$ & $225.50 \pm 43.03$ & $194.00 \pm 55.19$ & $0.000^{*}$ & $-.418^{\star \star}$ & 0.000 & $.392^{\star \star}$ & 0.000 \\
\hline $\begin{array}{l}\text { Dark } \\
\text { adaptation } 3.0 \\
\text { b-wave }\end{array}$ & $488.0 \pm 163.21$ & $437.52 \pm 67.03$ & $407.11 \pm 89.78$ & $0.025^{*}$ & $-.198^{*}$ & 0.033 & $.297^{\star \star}$ & 0.001 \\
\hline $\begin{array}{l}\text { Bright } \\
\text { adaptation[15] } \\
3.0 \text { a-wave }\end{array}$ & $57.83 \pm 22.17$ & $51.29 \pm 16.60$ & $41.61 \pm 11.03$ & $0.000^{*}$ & $-.433^{\star *}$ & 0.000 & $.414^{\star *}$ & 0.000 \\
\hline $\begin{array}{l}\text { Bright } \\
\text { adaptation } 3.0 \\
\text { b-wave }\end{array}$ & $152.01 \pm 53.34$ & $135.39 \pm 38.96$ & $105.44 \pm 28.46$ & $0.000^{\star}[16]$ & $-.445^{\star *}$ & 0.000 & $.345^{\star *}$ & 0.000 \\
\hline
\end{tabular}

**At 0.01 level (two-tailed), the correlation was significant.* At 0.05 level (two-tailedl), the correlation was significant.

Single-factor ANOVA test method for inter-group differences.

Table 5 Peak time of response waveform differences and correlation

with $\mathrm{AL}$ and $\mathrm{SE}$ in the F-ERG

\begin{tabular}{|c|c|c|c|c|c|c|c|c|}
\hline \multirow[t]{2}{*}{ Peak time (ms) } & \multirow{2}{*}{$\begin{array}{l}\text { High } \\
\text { myopia } \\
\text { group }\end{array}$} & \multirow{2}{*}{$\begin{array}{l}\text { Ultra-high } \\
\text { myopia } \\
\text { group }\end{array}$} & \multirow[t]{2}{*}{$\begin{array}{l}\text { Extremely high } \\
\text { myopia group }\end{array}$} & \multirow[t]{2}{*}{$\mathrm{P}$} & \multicolumn{2}{|c|}{$\begin{array}{l}\text { Relationship } \\
\text { with } A L\end{array}$} & \multicolumn{2}{|c|}{$\begin{array}{l}\text { Relationship } \\
\text { with SE }\end{array}$} \\
\hline & & & & & $\mathrm{R}$ & $\mathrm{P}$ & $\mathrm{R}$ & $\mathrm{P}$ \\
\hline $\begin{array}{l}\text { Dark } \\
\text { adaptation } 3.0 \\
\text { a-wave }\end{array}$ & $16.34 \pm 1.23$ & $16.19 \pm 1.35$ & $17.12 \pm 1.17$ & $0.000^{*}[17]$ & $-.418^{\star \star}$ & 0.000 & $.392^{\star \star}$ & 0.000 \\
\hline $\begin{array}{l}\text { Dark } \\
\text { adaptation } 3.0 \\
\text { b-wave }\end{array}$ & $42.88 \pm 5.08$ & $44.38 \pm 4.80$ & $45.07 \pm 4.99$ & 0.102 & $-.198^{*}$ & 0.033 & $.297^{\star *}$ & 0.001 \\
\hline $\begin{array}{l}\text { Bright } \\
\text { adaptation } 3.0 \\
\text { a-wave }\end{array}$ & $14.90 \pm 1.40$ & $15.00 \pm 1.04$ & $15.21 \pm 1.30$ & 0.629 & $-.433^{\star \star}$ & 0.000 & $.414^{\star *}$ & 0.000 \\
\hline $\begin{array}{l}\text { Bright } \\
\text { adaptation } 3.0 \\
\text { b-wave }\end{array}$ & $31.72 \pm 0.89$ & $31.52 \pm 1.27$ & $32.07 \pm 1.20$ & 0.099 & $-.445^{* *}$ & 0.000 & $.345^{\star \star}$ & 0.000 \\
\hline
\end{tabular}

**At 0.01 level (double tail), the correlation is significant. *At 0.05 level (double tail), the correlation is significant. Singlefactor ANOVA test method for inter-group differences.

The line fit comparative analysis showed that $\mathrm{AL}$ and SE correlated with the dark adaptation 3.0 a-wave and b-wave and bright adaptation 3.0 a-wave and b-wave. When the AL increased by $1 \mathrm{~mm}$, the dark adaptation 3.0 a-wave amplitude decreased by $21.63 \mathrm{uV}$, the light adaptation 3.0 a-wave amplitude reduced by $4.99 \mathrm{uV}$, the dark adaptation 3.0 b-wave amplitude reduced by $15.84 \mathrm{uV}$, and the light adaptation $3.0 \mathrm{~b}$-wave amplitude decreased by 13[18] .99 uV. An increase in SE of -1D was associated with a dark adaptation 3.0 a-wave amplitude reduction of $11.76 \mathrm{uV}$, a light adaptation 3.0 awave amplitude reduction of $2.36 \mathrm{uV}$, a dark adaptation $3.0 \mathrm{~b}$-wave amplitude reduction of $12.75 \mathrm{uV}$, and a light adaptation 3.0 b-wave amplitude reduction of 5.83uV (Figure 2).

\section{Correlation of Microvascular Morphology and Function in Macular Area}


According to Pearson's correlation analysis, the foveal thickness was significantly and positively correlated with the deep and superficial foveal blood flow densities and negatively correlated with FAZ (all P < 0.001) (Figure 3). The maximum comprehensive response a-wave and bright adaptation a-wave and b-wave amplitudes correlated negatively with the blood flow density (shallow and deep layer) at the fovea $(P<0.05)$ (Figure 4$)$ and positively with FAZ $(P<0.05)$. However, the OP2 wave amplitude and OP wave group sum average amplitude were positively correlated with the overall blood flow density of the superficial retina and the superficial parafovea and the blood flow density surrounding the superficial fovea $(P<0.05)$. The OP wave group amplitude was significantly and positively correlated with the above-mentioned blood flow densities $(P<0.01)$ (Figure 4).

\section{Discussion}

\section{Changes in macular microvascular morphology of high myopia}

The microvascular system of the retina is the foundation of visual function. In the past, research on the characteristics of retinal blood vessels was mostly conducted via fundus fluorescein angiography (FFA[1]) examination, which is an invasive operation that cannot visualize the deep retinal capillary network well, and it is difficult to quantify the retinal blood vessels. In contrast, OCTA has the advantages of non-invasiveness, rapid imaging, high resolution, and good reproducibility and has been widely used in studies of various fundus diseases. In this study, OCTA was used to quantitatively analyze the retinal microvascular morphology of the macular area of patients with high myopia. We found that, as the degree of myopia worsens, and the axial length of the eye increases, the FAZ area tends to decrease and the foveal retinal thickness appears to increase.

Tan et al. [15] conducted a study on 117 adults, including those with high myopia, and found the FAZ area to be negatively correlated with the eye axis and positively correlated with the myopia SE. The FAZ of highly myopic eyes was significantly and negatively correlated with the macula foveal retinal thickness. [2] Using OCTA, Samara et al.[16] also found that the FAZ area and macular foveal retinal thickness were negatively correlated. Similarly, Tick et al.[17] found there was a significant and negative correlation between the FAZ area and macular foveal retinal thickness, which may be related to the macular foveal retinal thickness[3] . The shape of the macular fovea [4] is also correlated, the deeper the macular depression, the wider the superficial retinal separation and, thus, the larger the FAZ area. The myopic FAZ area results of this study are quite different. Cheng et al.[18] and Min et al. [19] found that the FAZ area of highly myopic eyes was larger than that of emmetropic eyes, and it was positively correlated with the eye axis, which is contrary to our research results. However, Ucak et al. [20] believed that the FAZ area of high myopia patients does not correlate with AL [5] or SE. Studies have found that the FAZ shape and area are highly valuable indicators for monitoring, early diagnosis, treatment [21], and visual functional rehabilitation and follow-up of retinal vascular diseases, such as diabetic retinopathy, branch retinal vein occlusion, and other conditions with increased FAZ area. However, the results of research into the patterns and mechanisms of FAZ area changes in high myopia are inconclusive[6] . During the process of high myopic axial lengthening, the eyeballs do not uniformly expand to maintain the spherical shape [22]; the difference in the length of the high myopia axis leads to different degrees of optical magnification, and current OCTA equipment does not provide correction methods[23]. This may affect the accuracy of the FAZ area of highly myopic eyes, resulting in discrepancies.

The blood flow density of the macular fovea and parafovea at the superficial and deep retina levels, as measured by OCTA, showed that, with the deepening of myopia and the extension of $A L$, the blood flow density of the macular fovea in high myopia significantly increased, and the blood flow density of superficial and deep macular fovea significantly decreased. There have been numerous studies on the change characteristics of blood vessel density in the macular area of highly myopic eyes, but the results obtained are quite diverse. Yang et al. [24] and Li et al. [25] measured and analyzed the microvascular density of the parafoveal superficial layer, deep retina, and overall retina of high myopia and found them to

Page $8 / 18$ 
be significantly reduced compared with emmetropia and negatively correlated with AL. When Cheng [18] et al. evaluated the capillary loss of 145 subjects with refractive errors, they found that the blood vessel density of the superficial and deep parafovea of high myopia decreased, a change that was related to AL. The deep central fovea blood flow density is most closely related to the loss of retinal capillaries related to myopia and can be used as a sensitive indicator for detecting capillary loss in high myopia. Min et al. [19] found that parafoveal vessel density was decreased and the superficial capillary plexus was significantly reduced in patients with high myopia without pathological changes, while there was no significant difference in the deep parafoveal vessel density[7] . In a prospective study, Venkatesh et al. [26] found that deep blood vessel density changes in the macular area of simple high myopia were positively correlated with AL and negatively correlated with myopic SE, while the superficial blood vessel density was not significantly correlated with AL or myopic SE. We speculated that the difference in the thickness of each retinal layer and the division rules of OCTA for the superficial retinal capillary layer and deep retinal capillary layer may have led to differences in the macular blood vessel density measurements in the above studies.

According to our study findings, the FAZ area decreased with the increase in myopia degree and the extension of the ocular axis, while the total retinal thickness and the blood flow density in the foveal area[8] increased, and the vascular density in the parafovea and surrounding area decreased. These signs may present before the occurrence of myopic macular disease in high myopia and the decline in best-corrected visual acuity, and the central macular area ensures the normal microcirculation of the macular fovea by reducing the FAZ area, thickening the fovea retinal thickness, and increasing the vascular density to compensate. This compensation provides adequate oxygen and nutrients for the retina at the fovea to ensure the normal function of the optic cells in the central macular area. The decrease in vascular density near and around the fovea may be due to the increase in the eye axis, dilatation of the posterior eyeball wall, and mechanical pulling of the retinal vessels, which lead to thinning of the blood vessels, decreased blood flow, and decreased vascular density. Some studies have shown that the shape of the retinal vascular network is affected by neuronal metabolic demand during the development of the macular fovea. The thickness of the retinal ganglion cell layer is significantly correlated with vascular density [27-28]. With increasing age or extension of the eye $\mathrm{AL}$, the retinal ganglion cell layer thins and vascular density decreases, but there are fewer retinal ganglion cells in the macular fovea and the morphology is stable [29]. The vascular density does not decrease, but the compensatory increase can also explain our experimental results.

\section{Changes in visual function of F-ERG in high myopia}

The a-wave of F-ERG is the photoreceptor potential, which depends on the strength and integrity of the photoreceptor. The b-wave originates from the inner retinal layer and depends on the integrity of the signal transmission process in the awave. The OP is a positive wave attached to the b-wave, and it mainly reflects the function of amacrine cells, etc.; it is closely related to the activity of the inner layer of the retina and is a sensitive indicator of blood circulation in the retina. Our results showed that the scotopic, bright-adapted ERG a-wave and b-wave, OP wave group, and $30 \mathrm{~Hz}$ scintillation light P1 amplitudes of the three groups of highly myopic people followed a downward trend as the eye axis increased in length and myopia worsened. In terms of the peak time of each response waveform, the differences between the peak times of the maximum comprehensive response a-wave and the peak times of OP2 were statistically significant among the groups; furthermore, there was no obvious correlation with the axis of the eye, and it[9] negatively correlated with the myopic lens spherical equivalent. With the increase in the degree of myopia, the peak time increased. However, differences in the peak times of the other waveforms were not statistically significant, and this is consistent with previous research reports. Sachidanandam et al.[6] found that, in the absence of signs of pathological myopic retinopathy, the F-ERG amplitudes linearly decreased with increasing eye AL. When the AL was greater than $28.0 \mathrm{~mm}$, Kander[13] discovered that the ERG values of highly myopic eyes and emmetropic eyes were significantly different; the b-wave amplitude was reduced and the latency was delayed in the highly myopic eyes[10] , while the F-ERG values of mild to moderate myopia and emmetropia were not significantly divergent. Sexual difference. The b-wave amplitude of the pathological myopia ERG was significantly reduced. [11] In the studies of Koh et al. [30] and Sachidanandam et al.[14], compared with the photopic 
response, the b-wave amplitude of high myopia and scotopic vision was lower and related to the size of the eye axis, and it was speculated that this might lead to early vision. [12] In addition, the function of the rod cells and, consequently, the activity of bipolar cells, was also affected. Recently, Wan et al. [31] extracted and analyzed simple cone and rod cell-driven ERG a-and b-waveforms, particularly the OP waveform, with standard ERG and found no pathological changes in the fundus of 42 cases. [13] In the young myopia patients included in this research[14] , the peak frequency of rod cell-driven $\mathrm{OP}$ and the amplitude of dark-adapted ERG a- and b-waves were positively correlated with the spherical equivalent of the lens, and there was no correlation between bright-adapted ERG a- and b-waves. This confirmed that the rod system functionally changes during the development of myopia, while the function of the cone system is not significantly affected, which also explains why patients with high myopia have poor scotopic vision despite displaying no obvious pathological changes to the fundus. [15] In addition to the increase in the eye axis and refractive errors in adults with high myopia, there is a reduction in the amplitude of each waveform of the ERG, and the potential ERG changes may be a result of several factors. Optically, due to the extension of the eye axis, the light path increases, resulting in retinal illumination. Because of increased eye $A L$, the distance between the ERG measuring electrode and the retina increases, and the reactivity of the retinal cells is generally reduced, resulting in a weakening of the ERG response. Factors related to the retina itself, including changes in the microstructure of the retina caused by high myopia, such as reduced photoreceptor density, abnormal photoreceptor function, etc., will also affect its response[16] .

\section{Correlation of macular microcirculation, macular structure, and electrophysiological function in people with high myopia}

In our study, the MRT [17] was significantly and positively correlated with the superficial and deep FVD [18] (both P < 0.001). However, there was no obvious correlation between MRT and the ERG electrophysiological parameters. The [19] adaptive a-wave and b-wave amplitudes were negatively correlated with the FVD, while most of the waveform peak times positively correlated with FVD. The larger the FVD, the lower the amplitude of the a- and b-wave adaptations, and the longer the peak times of most waveforms. Because FVD is positively correlated with eye AL, in people without obvious pathological high myopia, the higher the FVD, the heavier the compensatory foveal blood flow in high myopia, i.e., as the degree of myopia increases, the eye axis length increases, and the corresponding bright adaptation and amplitudes of the a- and b-waves are reduced and the peak time is prolonged[20]. Cone cells are, of course, concentrated in the fovea of the macula. In the absence of pathological abnormalities of the overall retinal structure, the light adaptation ERG largely represents the function of the focal cone cells, which can explain the light adaptation FVD and ERG [21] relationship to the a-wave and b-wave. However, our research did not find an obvious relationship between MRT and electrophysiology. We believe that the changes in the a-wave and b-wave amplitudes of the bright adapted retina of high myopia eyes negatively correlate with FVD, and the growth of the eye axis is a common influencing factor. The relationship between the two can also be analyzed via changes in the microstructure of the macula. Light adaptation at the center of the macula mainly depends on the thickness of the outer macular retina, especially the thickness of the photoreceptor cell layer. The FVD mainly depends on the thickness of the inner layer of the macula, especially the nerve fiber layer and ganglion cell layer.

Moreover, in our study, the OP2 wave amplitude and the average OP wave amplitude of the groups were positively correlated with the overall blood flow density of the superficial retina, superficial parafovea, and the area around the fovea. The inner layer of the retina is mainly composed of the nerve fiber layer and the ganglion cell layer. The OP wave[22] mainly reflects the condition of the microcirculation of the retinal inner layer. As the degree of myopia increases, the blood flow density of the superficial microcirculation decreases, the OP wave amplitude is reduced, and the function of the inner retina is weakened.

Our study evaluated the trends of change in the macular morphology and function of young, healthy people with different degrees of high myopia. We analyzed the three groups of high myopia people's macular FAZ, macular foveal retinal thickness, and macular microcirculation, as well as changes to the function of the retinal photoreceptor cells, and attempted to establish the existence of correlations between the morphological and functional indicators. We found that as the axis of the eye increased, the foveal retina thickness and foveal blood flow density increased, whereas the FAZ;

Page $10 / 18$ 
superficial parafoveal, deep parafoveal, and overall blood flow densities; the blood flow density of the area around the fovea; the a-wave, b-wave, OP wave, and scintillation amplitude of the ERG were reduced, and some peaks were prolonged. In the bright adaptation experiment, the [23] amplitudes of the a-wave and b-wave positively correlated with the FAZ and negatively correlated with the FVD. Lastly, the OP wave amplitude was positively correlated with the superficial macular blood flow density.

The present study had some shortcomings. First, we only examined the high myopia of young, healthy people aged 18-40, and some age bias is inevitable. Second, when exploring the correlation between the deep and superficial microcirculation of the retina and the functions of the inner and outer layers of the retina, the thicknesses of the inner and outer layers of the retina could also be analyzed to bridge the two parameters, and this was omitted. Third, this study only analyzed indicators of retinal microcirculation in the macular area, without statistical analysis of choroidal thickness or blood flow density, and we failed to inspect the correlation between macular microcirculation, macular structure, and electrophysiological function. Fourth, the OCTA retinal thickness and blood flow density data used in the analyses were all automatically obtained by the device, and the AL was not corrected for high myopia, which means the results may be slightly erroneous. Therefore, future research needs to optimize the inspection equipment procedures, use a combination of several inspection methods, adopt more relevant parameters, and include a larger sample size to facilitate a comprehensive analysis of the correlation between macular microcirculation, structure, and electrophysiological function.

\section{Conclusion}

As the degree of myopia and axis of the eye increases, the thickness of the retina in the center of the macula and macular foveal blood flow density increase, while the FAZ; overall blood flow density; blood flow densities of the superficial parafovea, parafovea, central deep retina, and area surrounding the fovea; and the a-wave, b-wave, OP wave, and scintillation light ERG amplitudes decrease; while some amplitude peaks are prolonged. In the progression of high myopia, the changes related to retinal dark adaptation are more sensitive than those related to bright adaptation, indicating that changes to the rod cells of the high myopic eye may occur earlier than changes to cone cells. As the axis of the eye expands, the cone cell function shows a downward trend, and components of the inner layer of the retina, such as the microvascular circulation and amacrine cells, are affected. High myopia patients may present subtle changes to the morphology and function of the macular area before significant pathological changes or central vision damage occur. The combined use of OCTA and F-ERG can help in the early diagnosis, condition monitoring, clinical treatment, and advisement of people at a high risk of myopia.

\section{Declarations}

\section{Ethics approval and consent to participate}

The study protocol adhered to the tenets of the Declaration of Helsinki and was approved by the institutional review board of the Changsha Aier Ophthalmology Refractive Clinic. The participants were informed of the study objectives, and signed informed consent was obtained from all participants.

\section{Consent for publication}

I accept that my article is publicated in BMC Ophthalmology.

\section{Availability of data and material}

The results/data/figures and material in this article are all available for publication.

\section{Competing interests}

Page $11 / 18$ 
I declare that the authors have no competing interests as defined by BMC, or other interests that might be perceived to influence the results and/or discussion reported in this paper.

\section{Funding}

This work is supported by: National Natural Science Foundation of China (Grant No. 81670859,81970801 to XD), Hunan Province research and development plan funding projects in key areas (Grant No. 2020SK2133 to XD) $₫$ Science and Technology Foundation of Changsha, Hunan, China (Grant No. kh1801229 to XD), Natural Science Foundation of Hunan Province, China (Grant No. 2019JJ40001 to XD) andScience and TechnologyFoundation of Aier Eye Hospital Group, China (Grant No. AR1906D1هAM1906D2 to XD and Aier Glaucoma Research Institute)

\section{Authors' contributions}

Mengdan Cao collected the datas, wrote the main manuscript text . Jinxiang Luo prepared all figures and tables. Junyi Ouyang and Fen Nie collected the datas. All authors reviewed the manuscript.

\section{Acknowledgements}

My deepest gratitude goes first and foremost to Professor Xuanchu Duan , my supervisor, for his constant encouragement and guidance. He has walked me through all the stages of the writing of this thesis. Withouthis consistent and illuminating instruction, this thesis could not have reached its present form.

Second, I would like to express my heartfelt gratitude to Jinxiang Luo, who help me prepared all figures and tables. I am also greatly indebted to Junyi Ouyang and Fen Nie, who helped me a lot in collected the datas and modified the translation of the article.

Last my thanks would go to my beloved family for their loving considerations and great confidence in me all through these years. I also owe my sincere gratitude to the members of the Eye Research Center of Second Xiangya Hospital of Central South Universitys and my friends who gave me their help and time in listening to me and helping me work out my problems during the difficult course of the thesis.

\section{References}

1. RONG S S, CHEN L J, PANG C P.Myopia genetics the Asia Pacific perspective [J].Asia Pac J Ophthalmol(Phila), 2016, 5(4): 236-244.

2. Katz J, Tielsch JM, Sommer A. Prevalence and risk factorsfor refractive errors in an adult inner city population. Invest Ophthalmol Vis Sci 1997;38:334-40.

3. Wang Q, Klein BE, Klein R, Moss SE. Refractive status inthe Beaver Dam Eye Study. Invest Ophthalmol Vis Sci1994;35:4344-7.

4. Wong TY, Foster PJ, Hee J, et al. Prevalence and risk factors for refractive errors in adultChinese in Singapore. Invest Ophthalmol Vis Sci 2000;41:2486-94.

5. Saw SM, Gazzard G, Shih-Yen EC, Chua WH. Myopia andassociated pathological complications. Ophthalmic PhysiolOpt2005;25:381-91.

6. Hayashi K, Ohno-Matsui K, Shimada N, et al. Long-term pattern of progression of myopic maculopathy: a naturalhistory study. Ophthalmology 2010;117:1595-611.

7. Savastano MC, Minnella AM, Tamburrino A, Giovinco G, Ventre S, Falsini B. Differential vulnerability of retinal layers to early age-related macular degeneration: evidence by SD-OCT segmentation analysis. Invest Ophthalmol Vis Sci. 2014;55(1):560-6. 
8. Tan O, Li G, Lu AT, Varma R, Huang D. Advanced Imaging for Glaucoma Study Group. Mapping of macular substructures with optical coherence tomography for glaucoma diagnosis. Ophthalmology. 2008;115(6):949-56.

9. Ito Y, Nakamura M, Yamakoshi T, Lin J, Yatsuya H, Terasaki H. Reduction of inner retinal thickness in patients with autosomal dominant optic atrophy associated with OPA1 mutations. Invest Ophthalmol Vis Sci. 2007;48(9): 4079-86.

10. Verhoeven VJ, Wong KT, Buitendijk GH, et al. Visual consequences of refractive errors in the general population.[J] Ophthalmology 2015;122(1):101-109.

11. Wu Q, Chen $Q$, Lin B, et al. Relationships among retinal/choroidal thickness, retinal microvascular network and visual field in high myopia.[J].Acta Ophthalmol, 2020, 98: e709-e714.

12. TsuiChK, Yang B, Yu S, et al. The Relationship between Macular Vessel Density and Thickness with Light Sensitivity in Myopic Eyes.[J].Curr. Eye Res., 2019, 44: 1104-1111.

13. Kader MA, Electrophysiological study of myopia.[J].Saudi J Ophthalmol, 2012, 26: 91-9.

14. Sachidanandam R, Ravi P, Sen P. Effect of axial length on full-field and multifocal electroretinograms. [J] Clin Exp Optom. 2017;100(6):668-675.

15. Tan CS, Lim LW, Chow VS, et al. Optical Coherence Tomography Angiography Evaluation of the Parafoveal Vasculature and Its Relationship With Ocular Factors. [J] Invest Ophthalmol Vis Sci. 2016;57(9):OCT224-OCT234.

16. Samara WA, Say EA, Khoo CT, et al. CORRELATION OF FOVEAL AVASCULAR ZONE SIZE WITH FOVEAL MORPHOLOGY IN NORMAL EYES USING OPTICAL COHERENCE TOMOGRAPHY ANGIOGRAPHY. [J] Retina. 2015;35(11):2188-2195.

17. Tick S, Rossant F, Ghorbel I, et al. Foveal shape and structure in a normal population.[J].Invest. Ophthalmol. Vis. Sci., 52(8), 5105-10.

18. Cheng D, Chen Q, Wu Y, et al. Deep perifoveal vessel density as an indicator of capillary loss in high myopia. [J] Eye (Lond). 2019;33(12):1961-1968.

19. Min CH, Al-Qattan HM, Lee JY, et al. Macular Microvasculature in High Myopia without Pathologic Changes: An Optical Coherence Tomography Angiography Study. [J] Korean J Ophthalmol. 2020;34(2):106-112.

20. Ucak T, Icel E, Yilmaz H, et al. Alterations in optical coherence tomography angiography findings in patients with high myopia. [J] Eye (Lond). 2020;34(6):1129-1135.

21. Kwon J, Choi J, Shin JW, et al. Alterations of the foveal avascular zone measured by optical coherence tomography angiography in glaucoma patients with central visual field defects. Invest Ophthalmol Vis Sci 2017;58:1637-45.

22. Lee JH, Lee MW, Baek SK, Lee YH. Repeatability of Manual Measurement of Foveal Avascular Zone Area in Optical Coherence Tomography Angiography Images in High Myopia. Korean J Ophthalmol. 2020;34(2):113-120.

23. Fu D, Li M, Zeng L, Shang J, Yu Z, Zhou X. The role of magnification correction in macular vessel density assessment: a contralateral eye study in anisometropia patients. Ann Transl Med. 2021;9(5):380.

24. Yang $\mathrm{Y}$, Wang J, Jiang $\mathrm{H}$, et al. Retinal Microvasculature Alteration in High Myopia. [J] Invest Ophthalmol Vis Sci. 2016;57(14):6020-6030.

25. Li M, Yang Y, Jiang H, et al. Retinal Microvascular Network and Microcirculation Assessments in High Myopia. [J]. Am J Ophthalmol. 2017; 174:56-67.

26. Venkatesh R, Sinha S, Gangadharaiah D, et al. Retinal structural-vascular-functional relationship using optical coherence tomography and optical coherence tomography - angiography in myopia. [J]. Eye Vis (Lond). 2019; 6:8. Published 2019 Mar 7

27. Joyal JS, Gantner ML, Smith LEH. Retinal energy demands control vascular supply of the retina in development and disease: The role of neuronal lipid and glucose metabolism. [J]. Prog Retin Eye Res. 2018;64:131-156.

28. Kim K, Kim ES, Yu SY. Optical coherence tomography angiography analysis of foveal microvascular changes and inner retinal layer thinning in patients with diabetes. [J]. Br J Ophthalmol. 2018;102(9):1226-1231. 
29. Qu D, Lin Y, Jiang H, et al. Retinal nerve fiber layer (RNFL) integrity and its relations to retinal microvasculature and microcirculation in myopic eyes. Eye Vis (Lond). 2018;5:25.

30. Koh V, Tan C, Nah G, et al. Correlation of structural and electrophysiological changes in the retina of young high myopes. [J].OphthalmicPhysiol Opt. 2014;34(6):658-666.

31. Wan W, Chen Z, Lei B. Increase in electroretinogram rod-driven peak frequency of oscillatory potentials and darkadapted responses in a cohort of myopia patients. Doc Ophthalmol. 2020;140(2):189-199.

\section{Figures}

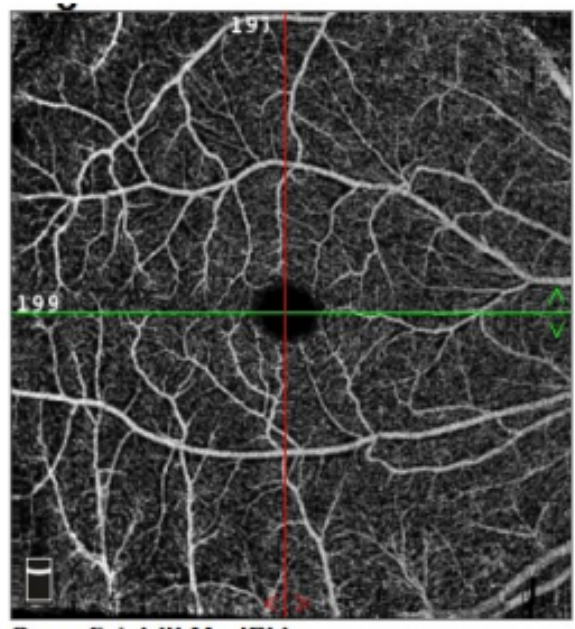

Superficial (ILM - IPL)

(a)

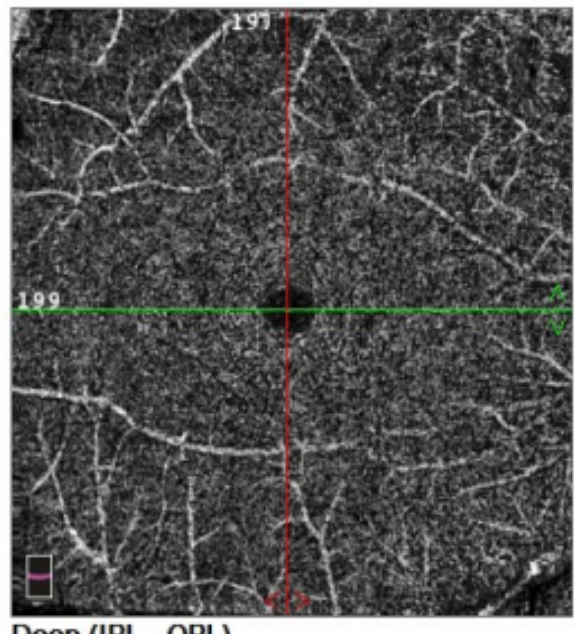

(b)

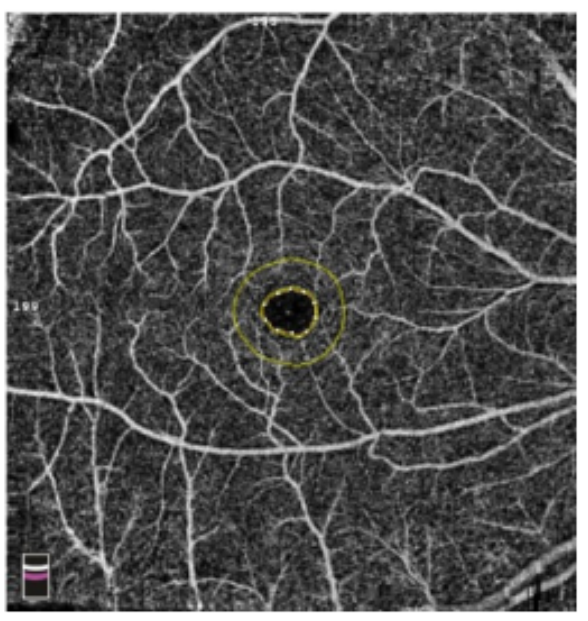

(c)

\section{Figure 1}

OCTA "retina-scan" mode. (a-c) OCTA analysis software automatically divided the macular area retinal image into the superficial capillary network (superficial) and deep capillary network (deep) and measured the foveal avascular area. 


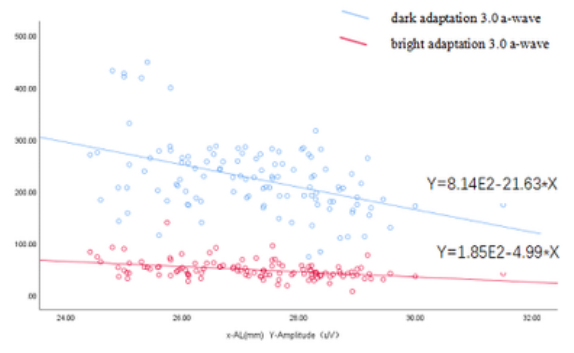

(a)

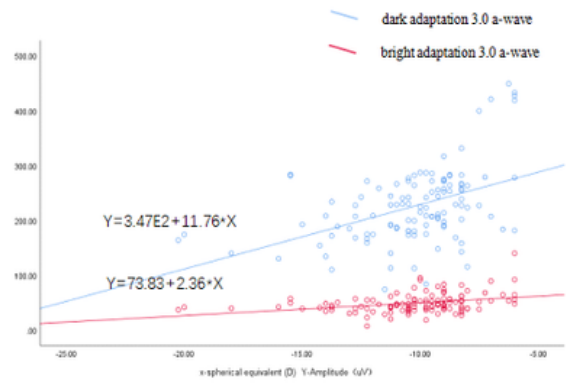

(b)

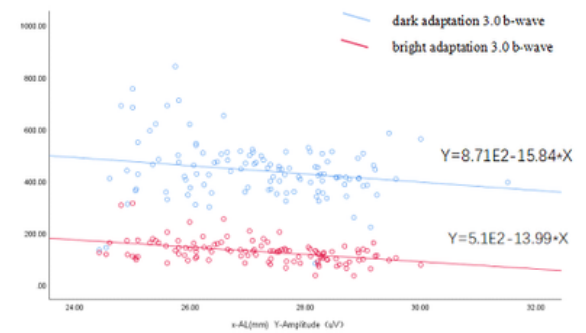

(c)

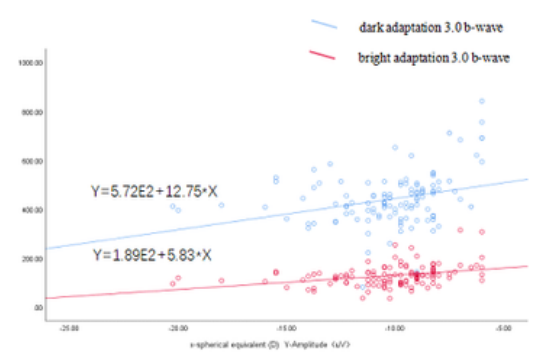

(d)

\section{Figure 2}

Line fit comparative analysis of the correlation between dark adaptation 3.0 a-wave and b-wave, bright adaptation 3.0 awave and b-wave with axial length and spherical equivalent. (a-d) a: Linear fitting relationship between a-wave and axial length of max-synthetic reaction; b: Linear fitting relationship between b-wave and eye axis of max-synthetic reaction; c: Linear fitting relationship between a-wave and spherical equivalent for maximum synthesis reaction and 3.0 wave; $d$ : Linear fitting relationship between b-wave and spherical equivalent for maximum synthesis reaction and 3.0 wave 


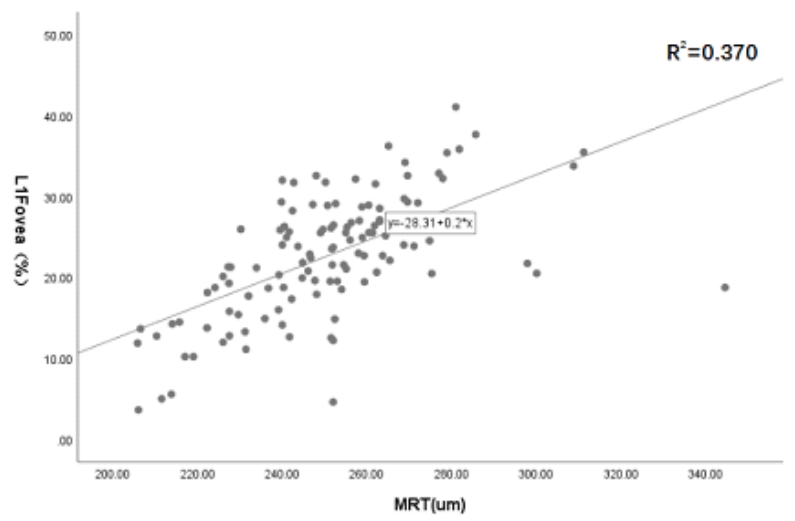

(a)

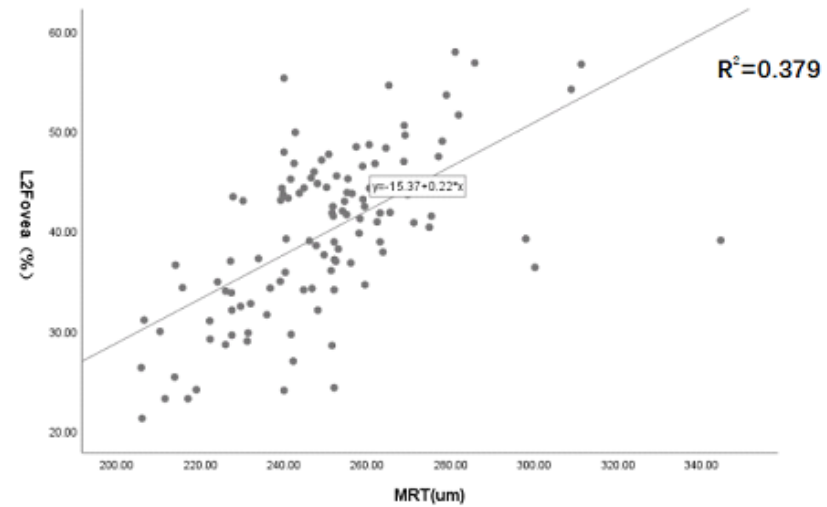

(b)

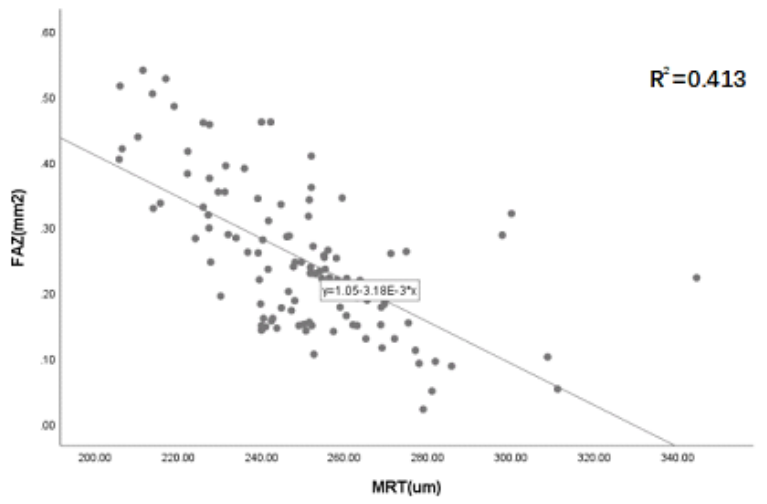

(c)

\section{Figure 3}

Pearson correlation analysis of MRT, FVD, and FAZ. (a-c) a: Correlation between MRT and superficial FVD; b: Correlation between MRT and deep FVD; c: Correlation between MRT and FAZ. MRT represents the thickness of the retina in the fovea, FVD is the density of foveal blood flow, and FAZ is the area of the foveal avascular zone. 


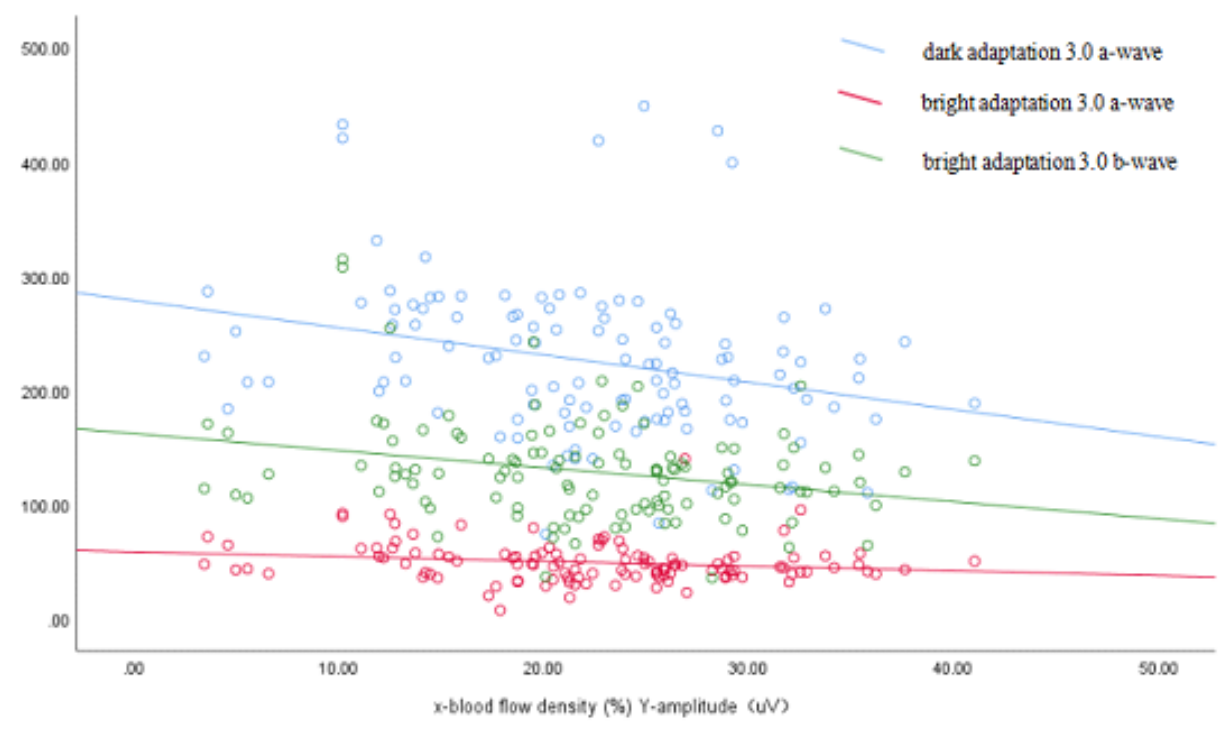

(a)

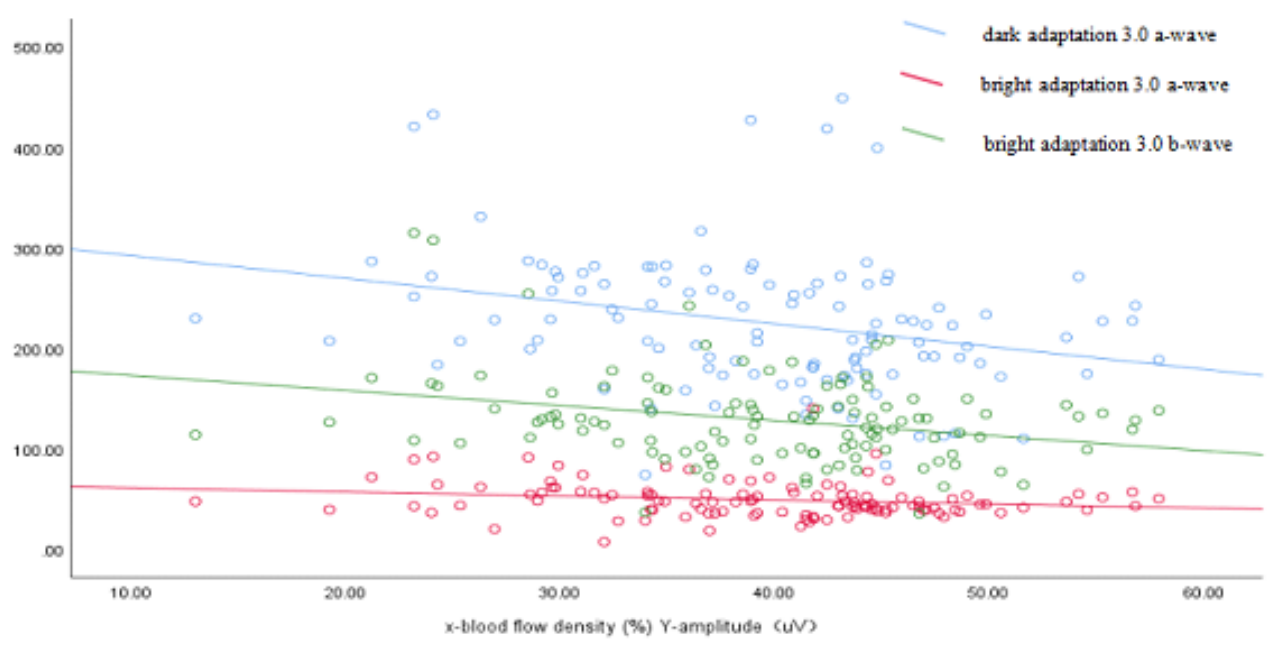

(b)

Figure 4

Correlation between amplitude and blood flow density of fovea in the maximum synthesis reaction. (a-b) a: Correlation between amplitude of a-wave, adaptive a-wave and b-wave, and superficial fovea in the maximum synthesis reaction; $b$ : Correlation between amplitude of a-wave, adaptive a-wave and b-wave, and deep fovea in the maximum synthesis reaction. X: blood flow density (\%); Y: amplitude (uV). 


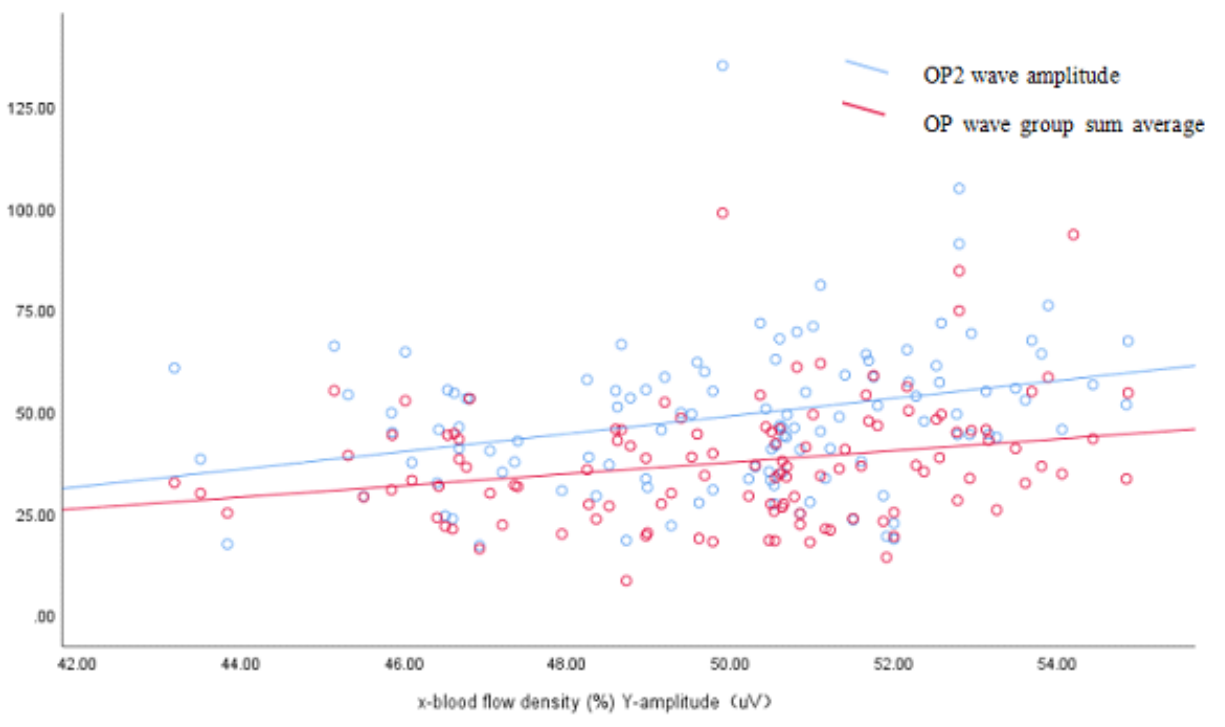

(a)

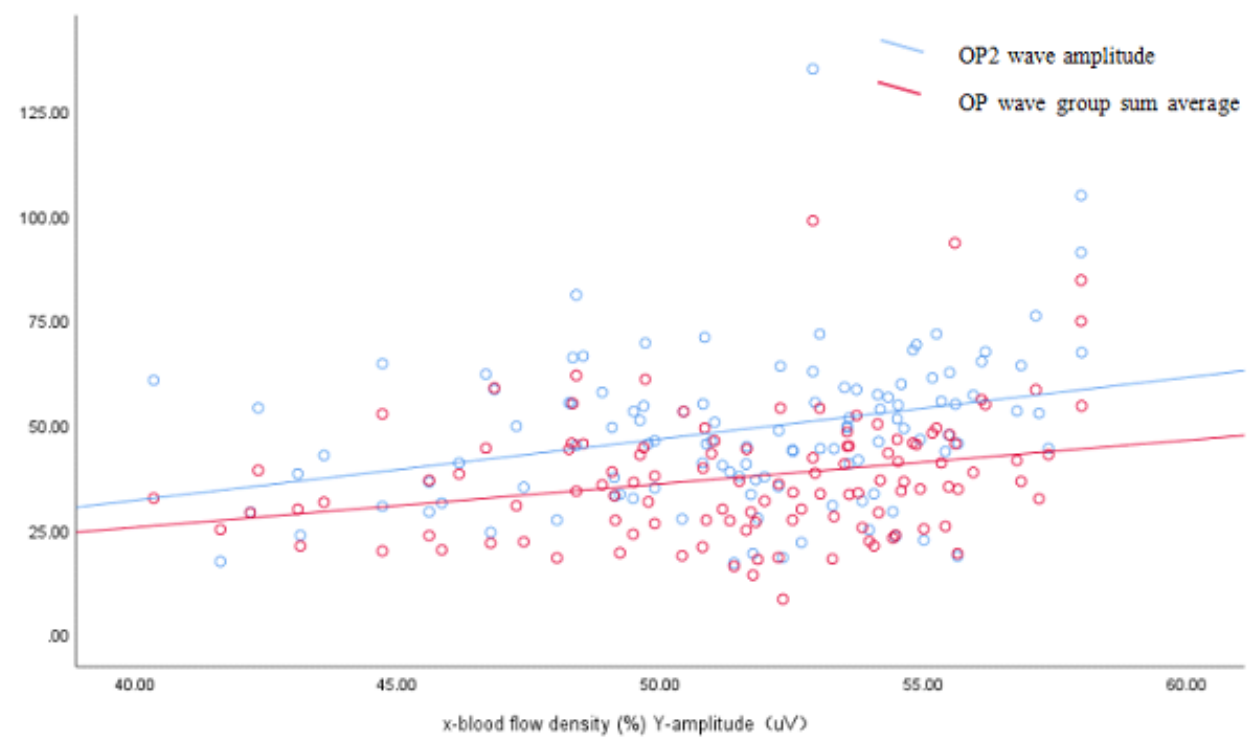

(b)

\section{Figure 5}

The correlation between mean amplitude and blood flow density of macular area. (a-b) a: Correlation between mean amplitude of OP2 and OP waves and total blood flow density of superficial macular retina; b: Correlation between mean amplitude of OP2 and OP waves and superficial parafovea retinal blood flow density. X: blood flow density (\%); Y: amplitude (uV) 